\title{
Pour une critique de la représentation
} tridimensionnelle des architectures mégalithiques en Europe occidentale. Méthodes et usages actuels

A critique of the 3-D representation of megalithic architecture in western

Europe: methods and current uses

\section{Serge Cassen, Laurent Lescop et Valentin Grimaud}

\section{(2) OpenEdition \\ Journals}

Édition électronique

URL : http://journals.openedition.org/abpo/2559

DOI : 10.4000/abpo.2559

ISBN : $978-2-7535-2782-9$

ISSN : 2108-6443

\section{Éditeur}

Presses universitaires de Rennes

\section{Édition imprimée}

Date de publication : 30 mars 2013

Pagination : 7-31

ISBN : 978-2-7535-2780-5

ISSN : 0399-0826

\section{Référence électronique}

Serge Cassen, Laurent Lescop et Valentin Grimaud, «Pour une critique de la représentation tridimensionnelle des architectures mégalithiques en Europe occidentale. Méthodes et usages actuels », Annales de Bretagne et des Pays de l'Ouest [En ligne], 120-1 | 2013, mis en ligne le 30 mars 2015, consulté le 19 avril 2019. URL : http://journals.openedition.org/abpo/2559; DOI : 10.4000/ abpo.2559 


\title{
Pour une critique de la représentation tridimensionnelle des architectures mégalithiques en Europe occidentale Méthodes et usages actuels
}

\author{
Serge CASSEN \\ CNRS, Laboratoire de recherches archéologiques (LARA) \\ Université de Nantes, 44312 Nantes (serge.cassen@univ-nantes.fr)
}

Laurent LESCOP

École nationale supérieure d'architecture de Nantes, Laboratoire GERSA (Groupe d'étude et de recherche scénologique en architecture) - 6 quai François-Mitterrand, 44262 Nantes (laurent.lescop@nantes.archi.fr)

Valentin GRIMAUD

Doctorant LARA et GERSA (valentin.grimaud@univ-nantes.fr)

Architecture mégalithique est une expression commode partagée par les archéologues de la Préhistoire récente de l'Europe. Il s'agit bien d'architecture puisqu'au-delà de cet art, de cette science et de ces techniques de la construction, par métonymie le vocable désigne l'ensemble d'un édifice et ses caractères architecturaux typiques. Et l'adjectif mégalithique s'écarte assez du Mégalithisme, désignant comme tous les mots en "isme " un concept idéologiquement connoté qui conduit le plus souvent à en faire l'économie, pour laisser entendre que le rocher, lourd par essence, prime bien dans la construction.

L'expression recouvre cependant une variété d'ouvrages qui va des tombes aux stèles, que la pierre soit brute ou mise en forme, qu'elle pèse plusieurs dizaines de tonnes ou se limite au poids d'un moellon aisément manipulable dont seule l'accumulation en proportion extraordinaire a permis la préservation de l'ensemble au-dessus du sol, en défiant le temps de l'érosion, un des buts recherchés. La nature de ces vestiges est par conséquent exceptionnelle, puisque les élévations en archéologie préhistorique sont d'une extrême rareté, l'essentiel étant enfoui, presque toujours invisible, souvent en raison de matériaux moins durs, moins volumineux, et de destructions conséquentes au cours du temps. 
La représentation de ces vestiges dépend déjà d'une longue histoire, dont nous ne ferons pas ici le bilan. L'objectif de cet article est plutôt de rendre compte de certains moyens que désormais nous jugeons utiles d'employer pour décrire et analyser non seulement un immeuble mais encore les signes gravés et peints à sa surface, qui livrent un accès privilégié à la pensée des $\mathrm{V}^{\mathrm{e}}$ et $\mathrm{IV}^{\mathrm{e}}$ millénaires av. J.-C. La réflexion menée sur les diverses acquisitions des données spatiales et historiques nous donnera cependant l'occasion de revenir par la critique sur une question inhérente à la représentation, qui est celle de la restauration. La Bretagne est, à cet égard, une terre d'élection des objets architecturaux si spécifiques que sont " dolmens " et "menhirs", et de leur mise en scène ${ }^{1}$. Pour illustration nous évoquerons les expériences actuelles, qui courent de la technique à la sémantique, notamment au sein des monuments de Gavrinis et de la Table des Marchands en Morbihan.

\section{La représentation en préambule}

Question d'espaces. Pour H. Poincaré, le cadre de nos représentations et de nos sensations pourrait être appelé l'espace représentatif. Nos représentations n'étant que la reproduction de nos sensations, elles ne peuvent donc se ranger que dans le même cadre. De même qu'un peintre ne peut figurer sur un tableau-plan des objets avec leurs trois dimensions, de même il est impossible de nous représenter les corps extérieurs dans l'espace géométrique.

L'espace représentatif, précise le philosophe des sciences, n'est qu'une image de l'espace géométrique, image déformée par une sorte de perspective, et les objets seront représentés en les pliant aux lois de cette perspective. "Nous ne nous représentons donc pas les corps extérieurs dans l'espace géométrique, mais nous raisonnons sur ces corps, comme s'ils étaient situés dans l'espace géométrique ${ }^{2}$. "Quand on dit, d'autre part, que nous " localisons" tel objet en tel point de l'espace, cela signifie que nous nous représentons les mouvements qu'il faut faire pour atteindre cet objet, nous nous représentons les sensations musculaires qui les accompagnent et qui n'ont aucun caractère géométrique.

Ce préambule aimerait ainsi faire valoir la tension que nous vivons dans l'étude entamée sur les monuments mégalithiques, entre l'expérience éprouvée de la pesanteur et l'analyse morphologique des parois, entre la dimension architectonique et géométrique de l'ouvrage et la stratégie de

1. Cette introduction à la restauration et à la représentation des monuments mégalithiques a fait l'objet d'une conférence (SC) lors du Seminar on Conservation Principles (Restoration and Documentation), tenu à Helsingore (Danemark), du 21 au 23 février 2011, à l'invitation de Anne Mette Rahbæk, directrice du Kulturstyrelsen (Heritage Agency of Denmark) au sein du ministère de la Culture (Copenhague), que nous remercions vivement pour son accueil.

2. Poincaré, Henri, La Science et l'hypothèse, Paris, Bibliothèque de Philosophie scientifique, 1902, (réed. 1917, p. 75), 304 p. 
conservation de l'objet. À cela s'ajoute une somme d'attendus sur lesquels il est bon de s'entendre.

Au-delà d'un objectif fondamental qui est la déconstruction du discours convenu sur Gavrinis et sa recomposition à travers une nouvelle grille de lecture des signes gravés, notre problématique actuelle est l'étude des processus informatiques et humains qui permettent la communication visuelle d'informations au travers d'une représentation tridimensionnelle. Mais également la compréhension ou l'interprétation de cette représentation par l'utilisateur.

Si l'information sous sa forme électronique est désormais acquise en routine au sein des laboratoires d'archéologie, les systèmes de traitement automatique ne sont pourtant pas toujours en mesure de permettre à l'archéologue d'en faire bon usage. Ce processus de traitement de l'information est d'autant plus difficile que la quantité d'informations et les modes de représentation proposés par le système ne sont pas nécessairement adaptés à ses propres stratégies de traitement. C'est la raison qui pousse aujourd'hui à cette collaboration entre géomètres, architectes et archéologues, de même que nous trouvons ces mêmes réflexions affichées par des structures de réflexion comme le Laboratoire d'informatique de Grenoble (P. Genoud) dans ses recherches sur les systèmes d'information spatio-temporelle (web sémantique, visualisation) et dans un domaine d'application privilégié (la prévention des risques naturels) en apparence fort éloigné de nos préoccupations du moment.

Prenons le mot visualisation. Il s'agit, pour nos collègues informaticiens, d'une transformation qui mène à la représentation visuellement perceptible d'une entité, transformation d'autant plus complexe que les informations à représenter n'ont pas nécessairement de transposition visuelle directe. Par ailleurs, la visualisation ne doit pas se limiter à produire des représentations visuelles de l'information, si ces dernières ne peuvent aussi être manipulées par l'utilisateur afin de comprendre et éventuellement d'interagir avec l'espace d'information. C'est bien cette étape qui nous a manqué pendant des années, nous le verrons brièvement plus loin, dans l'étude tridimensionnelle des dolmens du Morbihan par l'informatique...

La représentation visuelle d'informations au travers de scènes tridimensionnelles synthétisées s'inscrit dans la droite ligne des techniques de déformation dont parlait H. Poincaré. La déformation n'est autre que celle due à la perspective qui présente l'avantage d'être naturellement interprétée par l'homme cognitif, un effet de perspective si familier que notre système perceptif conduit à choisir une interprétation tridimensionnelle là où la troisième dimension n'existe pourtant pas (peinture en trompe-l'œil, etc.). Les images synthétisées utilisent par conséquent les mêmes artifices " picturaux " pour créer cette impression de relief : projection, perspective, lumière, ombres ou textures. Mais elles ont ceci de supérieur aux tableaux ou aux parois peintes, qu'une machine est cette fois capable de les recalculer dans une temporalité donnée. On passe alors d'une image fixe à une 
scène tridimensionnelle dans laquelle l'utilisateur peut " se déplacer "; en réalité, on le sait, il déplace le point de vue qu'il a de la scène. Cette possibilité pourra ainsi être exploitée pour permettre la compréhension de la structure interne ou externe d'un monument préhistorique.

En bref, la synthèse d'images tridimensionnelles est un ensemble de techniques permettant de figurer une géométrie spatiale à l'aide d'un ordinateur. Les données sont décrites dans un repère orthonormé $\mathrm{x}, \mathrm{y}$, z. Un objet est décomposé soit selon une trame régulière, soit selon une trame épousant au mieux les différentes inflexions de forme, l'ensemble des points, reliés en eux, décrivant faces et volumes. Il devient donc possible de produire des vues projetées ou perspectives. La première étape est donc la constitution de cet ensemble de points, étape nommée modélisation, qui peut être opérée à la main ou quasi automatiquement avec la lasergrammétrie ou la photogrammétrie. Le modèle est ensuite visualisé soit sous forme brute (on verra les points ou le maillage géométrique) soit sous forme " habillée " par l'ajout de textures, de lumières, et autres effets d'embellissement de l'image. Cette représentation aimerait enfin pouvoir s'affranchir de l'écran individuel de l'ordinateur pour être projetée dans un dispositif immersif collectif tel que nous le proposons pour comprendre l'agencement d'une tombe à couloir, espace idéal s'il en est pour y tester de tels dispositifs puisqu'elle est à la fois close en plan et en volume et cependant accessible par une entrée étroite et bien structurée.

\section{Un état de l'art}

En apparence mieux adaptée aux architectures antiques ou historiques (châteaux, cathédrales...) si l'on en croit une rapide enquête documentaire ainsi que la production audiovisuelle de ces vingt-cinq dernières années, et plutôt réservée en Préhistoire aux cavités souterraines et à leurs figurations peintes et gravées, la représentation tridimensionnelle appliquée aux monuments mégalithiques, malgré son évidence, est pourtant une préoccupation assez récente. Nous tenterons d'en faire un bref tour d'horizon (arrêté à l'automne 2012), en nous portant tout d'abord vers quelques expériences menées en Europe avant d'en venir à la France. Dans la mesure où les références publiées sont assez rares, force sera d'en passer par une webographie qui n'en demeure pas moins informative, et quoi qu'il en soit, le mode futur de nos échanges et de nos contrôles croisés ${ }^{3}$. Enfin, le lecteur ne trouvera pas ici un inventaire exhaustif des programmes menés sur le thème des mégalithes, mais plutôt un choix critique d'illustrations complémentaires d'intentions et de résultats.

3. Toutes les consultations des adresses URL (ressources sur Internet) ont été reprises et vérifiées entre le 10 et le 15 septembre 2012. 


\section{Portugal}

Le Museu de Arqueologia e Etnografia do Distrito de Setúbal est probablement la première institution européenne à avoir mené, en 1983, un levé photogrammétrique d'envergure sur un monument mégalithique de l'Alentejo près d'Évora, la gigantesque tombe à couloir de Anta Grande de Zambujeiro (fig. 1). Resté longtemps inédit, ce levé mené à l'aide d'images stéréoscopiques n'a été que récemment publié ${ }^{4}$. Il témoigne probablement des difficultés d'exploitation que ces données photographiques et graphiques pouvaient occasionner chez des archéologues, à une époque où les levés numériques étaient encore bien peu développés, sans même évoquer la puissance très relative des micro-ordinateurs d'un emploi encore sporadique.

Le site bien connu des pierres dressées d'Almendres, sur ce même territoire d'Évora, souvent comparé à des monuments de Bretagne, est actuellement présenté en 3D sur Internet à travers l'affichage "stéréo HTML5 " qui permet de regarder des vidéos en 3D sur You Tube. L'affichage stéréoscopique propose une image différente pour chaque œil. L'intérêt est cependant assez limité pour un usage archéologique de l'image, sinon comme élément de documentation ou test sur l'objet d'étude ${ }^{5}$.

Plus intéressante est l'approche adoptée par Pedro Alvim (Centro de História da Arte e Investigação Artística da Universidade de Évora) qui a récemment modélisé par photographies numériques l'ensemble des stèles néolithiques de Fontainhas (Mora), toujours dans l'Alentejo. Le logiciel employé n'est pas spécifié sur le site Internet Academia. edu. On remarque avec intérêt les traces bien visibles de la mise en forme du monolithe central, en tournant autour de l'objet ${ }^{6}$.

\section{Espagne}

Un monument emblématique du nord-ouest de la péninsule Ibérique, la tombe à couloir de Dombate en Galice, a fait l'objet d'explorations il y a plus de 20 ans par J.M. Bello Diéguez. Laissé un peu à l'écart, ce monument a fait l'objet de restaurations récentes financées par le gouvernement régional et la présentation publique en est entièrement renouvelée. Un levé au scanner 3D en 2008 a débouché sur un tableau de mesures et quelques images (plans, sections) qui semblent n'avoir pas encore été exploitées par les archéologues ${ }^{7}$. Un centre d'interprétation - tout juste inauguré au mois

4. Soares, Joaquina, Tavaraes da Silva, Carlos, "Anta Grande do Zambujeiro. Arquitectura e poder, Intervençãoarqueológica do MAEDS, 1985-1987 ", Musa, Museus, Arqueologia e Outros Patrimónios, 3, 2010, p. 77-118.

5. [http://www.youtube.com/watch?v=rU7hJDLqu0U\&feature=player_detailpage].

6. [http://www.academia.edu/1473033/PDF_3D_work_in_progress_Fontainhas_standing_stone_enclosure_Mora_Portugal].

7. Cebriándel Moral, Fernando, Yáñez Rodríguez, J., Lestón Gómez, M., Vidal Pérez, F., CARrera RAmírez, F., El dolmen de Dombate, arqueología, restauración, arquitectura. A Coruña, Deputación da Coruña, 2011, 311 p. 
de juillet 2012 - utilise peu encore ces données tridimensionnelles dans la présentation classique, virtuelle ou recomposée du site.

Prolongement de cette restauration, une équipe entraînée par Natalia Cortón Noya et Fernando Carrera Ramírez a développé un enregistrement lasergrammétrique, photogrammétrique et orthophotographique exemplaire où les dommages menaçant les peintures et gravures des parois de la tombe à couloir (fig. 2) sont systématiquement inventoriés, localisées et commentées, qu'il s'agisse d'algues, lichens, champignons, rubéfaction, craquelures ou desquamations ${ }^{8}$. Une exposition du programme en cours vient d'être projeté à l'occasion de l'International Congress on Science and Technology for the Conservation of Cultural Heritage conduit au sein de l'université de Santiago de Compostela (octobre 2012) ${ }^{9}$.

Toujours en Galice, à Fonte Tourón Lalín (Pontevedra), la destruction d'un tertre néolithique a occasionné un levé 3D (scanner Leica HDS3000) d'une dalle gravée déplacée mais qui devait faire partie intégrante du monument originel. Du nuage de points traité sont extraites quelques sections du monolithe mais les gravures restituées ne semblent pas relever d'un traitement spécifique à partir des données spatiales ${ }^{10}$.

Autre expérience, deux stèles similaires de la région cantabrique ont été enregistrées dans des conditions fort dissemblables ${ }^{11}$. La première stèle du site de Sejos (longue de $3 \mathrm{~m}$ ), couchée en pleine montagne, a nécessité une acquisition sous tente de protection (lumière, climat) et l'aide de batteries; 40 scans ont été nécessaires à travers un dispositif SmartSCAN3D et un logiciel ArteCore de la firme Nepos. Quant à la stèle circulaire de Zurita, également datée du Chalcolithique et conservée au musée de Préhistoire de Santander ( $2 \mathrm{~m}$ de diamètre), elle a nécessité $16 \mathrm{scans}$ avec le même appareil pour mesurer une surface centrale de 1,5 $\mathrm{m}^{2}$. Les auteurs revendiquent dans les deux cas une meilleure lisibilité des objets (armes...) en vue d'une meilleure datation des gravures.

En nous portant maintenant vers l'extrémité méridionale de la péninsule, l'ensemble extraordinaire de Antequera a bénéficié récemment d'une mise en valeur et d'une médiatisation importante, notamment avec le monument de Menga refouillé en 2005 et dont un levé 3D au scanner Callidus CP3200

8. CARRERA RAMíREZ, Fernando, «El arteprehistórico y su conservación. Pinturas y grabados en Dombate ", dans Rodríguez, José Yáñez (dir.), El dolmen de Dombate : arqueología, arquitectura y conservación, A Coruña, Deputación da Coruña, 2011, p. 230-266.

9. [http://www.academia.edu/1891385/Non-invasive_recording_technologies_for_the_ study_and_conservation_of_prehistoric_rock_art_the_dolmen_of_Dombate].

10. Cabrejas Domínguez, Elena, Mañana-BorrazÁs, P., Seonne-Veiga Y., "Achado dunhas lousas con arteme galítico en Fonte Tourón Lalín, Pontevedra ", Cuadernos de estudios gallegos, LV, $\mathrm{n}^{\circ}$ 122, 2009, p. 9-31 [http://estudiosgallegos.revistas.csic.es/index.php/estudiosgallegos/issue/view/4].

11. Breuckmann, Bemd, Arias Cabal, Pablo, Mèlard, N., Ontanón, R., Pastoors, A., Mayolini, L.C., VegA, P., Weniger, G. C., Surface scanning - New Perspectives for Archaeological Data Management and Methodology? Computer Applications to Archaeology 2009 Williamsburg, Virginia, USA, 2009, 8 p. 
par F.J. Molero, dans le cadre d'un programme de recherche informatique de l'université de Granada (Automatic documenting of Cultural Heritage Sites from $3 D$ laser scanner data), permet de travailler sur un plan et des élévations précises. Aucune exploitation des données ne semble pour le moment publiée ${ }^{12}$.

Toujours en Andalousie, les dolmens de El Pozuelo 3 et 4 (province de Huelva) ont fait l'objet de fouilles récentes exemplaires par l'entreprise Cota Zero ${ }^{13}$. Un scanner temps-de-vol a été employé pour couvrir l'ensemble du site aménagé sur terrasses artificielles, auquel s'est ajouté une photogrammétrie aéroportée (par drone). La micro-topographie des lieux est obtenue en combinant un relevé par station totale et les nuages de points du scanner. Ce résultat est également comparé au même plan micro-topographique issu des données conjointes de la station totale et de la photogrammétrie. Ces essais méthodologiques sont, à notre connaissance, parmi les travaux les plus avancés en Europe relativement aux tombes à couloir néolithiques.

Notons enfin, dans le cadre d'une série télévisuelle bien connue en péninsule Ibérique ("Arqueomanía "), la récente diffusion (2011) d'un documentaire sur le dolmen I de Alberite, non loin de Cadiz, qui intègre plusieurs reconstitutions tridimensionnelles du monument fouillé dans les années $1990^{14}$.

\section{Angleterre}

En mars 2011, English Heritage, organisme souvent cité en exemple, communiquait dans la presse écrite et audiovisuelle ainsi que sur Internet à propos du monument de Stonehenge et du programme mêlant la méthode lasergrammétrique et la technologie d'imagerie numérique, afin de produire un modèle visuel et métrique le plus précis possible sur un site préhistorique mondialement réputé (" the world famous prehistoric monument ") ${ }^{15}$.

12. RuIZ GonzÁLEZ, Bartolomé, "El proyecto de tutela y valorización de los dólmenes de Antequera ", In : Dólmenes de Antequera. Tutela y ValorizaciónHoy, PH Cuadernos 23, Consejería de Cultura, Junta de Andalucía, 2009, p. 12-37.

13. Pallarés Silva, Victor, Linares Catela, D., Linares Catela, J. A., Lozano Martínez, F. R., BARRERA VERA, J.A., Topografía de vanguardia en los levantamientos de yacimientos megalíticos estrategias para el conocimiento. Métodos, dans 4th International Meeting on GraphicArchaeology and Informatics, Cultural Heritage and Innovation ARQUEOLÓGICA $2.0,20^{\text {th }}-22^{\text {th }}$ June 2012 Seville, à paraître.

14. Ramos Muñoz, José, Giles Pacheco, Francisco (eds.), El dolmen de Alberite (Villamartín), Aportaciones a las formas económicas y sociales de las comunidadesneolíticas en el noroeste de Cádiz, Cadiz, Universidad de Cadiz, servicios de publicaciones, 1996, 366 p. [http://www.rtve.es/alacarta/videos/arqueomania/arqueomania-capitulo-12/1306865/].

15. http://www.english-heritage.org.uk/about/news/stonehenge-in-high-definition/ (voir aussi la médiatisation du projet sur la BBC) : [http://www.bbc.co.uk/news/science-environment-12688085]. 
En septembre 2011, English Heritage annonçait que l'acquisition et le traitement des données scanner à Stonehenge était achevé ${ }^{16}$.

En 1993 déjà, un levé photogrammétrique avait permis d'obtenir un modèle satisfaisant, à $2 \mathrm{~cm}$ de précision. La résolution annoncée cette fois à $0,5 \mathrm{~mm}$ permettra une meilleure lecture des surfaces des monolithes, notamment avec le secret espoir de trouver de nouvelles informations sous la couverture des lichens. Graffiti modernes et gravures du Bronze ancien sont en effet connus, présupposant d'autres découvertes. La connaissance du processus d'érosion est un des objectifs que se fixe aussi English Heritage en mesurant les surfaces des monolithes plus ou moins altérées ${ }^{17}$. Apparaîtraient également les traces de dressage du grès (voir monolithe $\mathrm{n}^{\circ}$ 60, fig. 1), révélant le travail de différentes équipes (mais sans plus de détails).

La société savante Wessex Archaeology ${ }^{18}$ avait antérieurement enregistré les quelques gravures d'instruments en bronze gravés sur trois des monolithes du site ( ${ }^{\circ} 3,4$ et 53), en faisant appel à la firme Archaeoptics de Glasgow (scanner Minolta VI-900, à 8 pm de précision dans un champ limité à 0,7 x 2 m, à $1 \mathrm{~m}$ de distance). Très peu de signes distincts sont ici inventoriés (monotonie du motif de la hache métallique, tranchant vers le haut) et leur morphologie attendue ne pose aucun problème de reconnaissance. Tout dernièrement (oct. 2012), le quotidien The Independant révélait la découverte (faite sur les données acquises en 2011 par Greenhatch Group) de 72 nouvelles gravures de lames de haches métalliques datées du Bronze ancien sur un monolithe de Stonehenge ( $\mathrm{n}^{\circ} 4$, fig. 1 ), au moyen d'un enregistrement scanner et après plusieurs mois de travaux. 850 Go de données sont mobilisées (également photogrammétriques, acquises au moyen d'une grue mobile pour les sommets des monolithes) par ArcHeritage, une filiale de York Archaeological Trust ${ }^{19}$.

On appréciera la rapidité de divulgation du rapport par English Heritage ${ }^{20}$ qui permet de reconnaître les traces de mise en forme des monolithes et bien sûr ces nouvelles gravures. La résolution adoptée est de $1 \mathrm{~mm}$ pour les monolithes, et de 0,5 mm pour les surfaces gravées. Dans le même temps, pour tester le repérage de graffiti à moins de $0,5 \mathrm{~mm}$ de résolution, une solution photogrammétrique a été menée avec un instrument Leica V-LUX 2 Camera exploité avec le logiciel Agisoft PhotoScan. D'intéressantes appli-

16. [http://www.stonehengelaserscan.org/].

17. Field, David, Pearson, Trevor, Stonehenge World Heritage Site Landscape Project Stonehenge, Amesbury, Wiltshire, Archaeological Survey Report, Research Department Report Series 109, 2010, 86 p.

18. Goskar, Thomas, Carty, A., Cripps P., Brayne C., Vickers D., "The Stonehenge Lasershow ", British Archaeology 73, 2003.[http://www.britarch.ac.uk/ba/ba73/feat1.shtml]. 19. [http://www.independent.co.uk/news/science/archaeology/revealed-early-bronze-agecarvings-suggest-stonehenge-was-a-huge-prehistoric-art-gallery-8202812.html] .

20. ABBotT, Marcus, ANDERSON-WhYMARK, Hugo, Stonehenge laser scan : archaeological analysis report, Research report series $n^{\circ} 32$, English Heritage, Fort Cumberland, Portsmouth, 2012, $71 \mathrm{p}$. 
cations sont proposées autour de la mise en forme des monolithes et des enlèvements plus récents d'éclats-souvenirs (pour d'éventuels pèlerins). Une perspective est également évoquée pour renseigner l'usure provoquée par le passage des pieds des visiteurs sur les blocs accessibles ${ }^{21}$.

Une recomposition architecturale et environnementale du site est bien entendu la motivation plus évidente du gestionnaire du site en vue de sa (re)présentation au public. Un scanner Zoller+Fröhlich Imager 5010 est pour cela mis en œuvre, et l'université de Bournemouth, en collaboration avec Google Earth, a dernièrement présenté une carte virtuelle du site de Stonehenge dans son environnement néolithique ${ }^{22}$.

Moins méthodologique et s'en tenant plutôt à des intentions à venir, une étude de l'altération des gravures préhistoriques à l'air libre a fait l'objet d'un programme très attractif (Fading rock art lanscapes project) financé par la British Academy, conduit par le département d'archéologie de l'université de Durham en partenariat avec le département des sciences informatiques de l'université de Bristol $^{23}$. Une dalle gravée $(1 \mathrm{~m})$ de 60 cupules $(0,5$ à $1 \mathrm{~cm}$ de profondeur) a été numérisée par scanner Minolta 910 (8,6 millions de points), avec une précision infra-millimétrique ${ }^{24}$. Le projet affiché est bien de revenir chaque année pour mesurer le degré d'érosion, les chiffres obtenus en Scandinavie (une perte de matière de $6 \mathrm{~mm} / 1000$ ans sur granite) étant de nature à alarmer les conservateurs du patrimoine en plein air. Mais, depuis 2005, aucune autre information n'est à ce jour disponible pouvant éclairer le devenir de ce programme.

\section{Écosse}

En 2010, une équipe formée de conservateurs du patrimoine et d'experts en technologie numérique appartenant à Historic Scotland, au Glasgow School of Art et à la société CyArk, décide de numériser le " cour du Orkney néolithique ". Une sélection de sites réputés, conservés dans ces îles du nord-est de l'Écosse, a pu bénéficier de levés au scanner 3D à l'image de la tombe mégalithique de Maeshowe et des cercles de pierres dressées de Stenness et Ring of Brodgar, tous considérés comme appartenant au patrimoine mondial (Unesco World Heritage Site). Le programme est prévu se dérouler sur cinq années ${ }^{25}$.

Les premières vues présentées en promotion insistent sur les plans et sections spectaculaires de la tombe (avec son couloir d'accès; fig. 2), que rendent possibles ces nouvelles techniques d'enregistrement et de restitu-

21. [http://services.english-heritage.org.uk/ResearchReportsPdfs/032_2012web.pdf]

22. [http://www.bbc.co.uk/news/uk-england-dorset-16295843].

23. Barnett, Tertia, Chalmers, A., Díaz-Andreu, M., Ellis, G., Longhurst, P., SharPe, K., TrinKS, I., " 3D Laser Scanning For Recording and Monitoring Rock Art Erosion ", International Newsletter on Rock Art (INORA) 41, 2005, p. 25-29.

24. [http://www.dur.ac.uk/prehistoric.art/].

25. [http://www.orkneyjar.com/archaeology/2012/08/29/virtual-maeshowe-tour-goesonline/]. 
tion. La page Internet de l'entreprise CyArk permet d'ailleurs de se déplacer en temps réel dans le nuage de points acquis sur le site ${ }^{26}$.

Le programme envisage bien entendu d'acquérir des informations sur les rares gravures néolithiques parfois présentes au sein de ces monuments, mais également sur les signes runiques des marins vikings de passage dans l'archipel des Orcades, tout comme sur les graffiti des touristes de l'époque victorienne... Déjà en 2003 et 2004, plusieurs levés lasergrammétriques ponctuels avaient permis d'évaluer l'état des surfaces en rapport avec les processus d'altération (enlèvement ou dépôt de matière sur la roche). Le programme entamé en 2010 se déroule à plusieurs échelles d'acquisition, depuis le scanner aéroporté enregistrant le relief (précision du mètre) jusqu'aux capteurs allant chercher les fines incisions faites dans la roche (précision du millimètre). Le matériel employé, du plus précis vers le plus lointain, se décline selon les Konica Minolta VI9i, Leica HDS 6100 et Leica Scan Station 2. En août 2012, une brève visite virtuelle du site de Maeshowe est proposé sur la page de la revue en ligne Current Archaeology ${ }^{27}$.

Enfin, Pixogram, une société établie à Edinburgh, spécialisée en projets sur le patrimoine architectural (reconstruction et visualisation), propose dans son portfolio un cercle de pierres dressées et des stèles isolées (Tuilyies) enregistrés par photogrammétrie et replacés dans un environnement virtuel ${ }^{28}$. On note ici tout l'intérêt de pouvoir découvrir la météorisation visible sur la pierre en fonction des éclairages (fig. 2), une érosion opérant après l'érection du monolithe. Ces détails ne sont pas mentionnés par les opérateurs mais révèlent pour un spécialiste tout le potentiel de ces représentations.

\section{Irlande}

Dans la très fameuse vallée Bru na Boinne au nord de Dublin, le grand cairn de Knowth est bien connu pour les gravures conservées au sein de ces différentes tombes à couloir, ou visibles sur leur péristalithe à l'air libre. La tombe " satellite " $\mathrm{n}^{\circ} 14$ aux abords immédiats du grand monument principal a ainsi bénéficié en 2006 d'un levé au scanner ${ }^{29}$, s'inscrivant dans l'ambitieux programme national, Discovery Program, une institution publique fondée en 1991 pour la recherche archéologique promouvant les nouvelles technologies (scanner, lidar, prospections géophysiques, etc.).

Le matériel utilisé à Knowth est un scanner HD NextEngine (Model 2020i), piloté par Kippor Sinemaster 1000 et contrôlé par le logiciel Scanstudio HD PRO. Cette même instrumentation sera employée pour enregistrer les gravures d'un des orthostates décorés de la tombe à couloir de

26. [http://archive.cyark.org/maeshowe002-3dviewerpro].

27. [http://www.archaeology.co.uk/articles/news/chamber-of-secrets-historic-scotlandlaunches-virtual-tour-of-maeshowe.htm].

28. [http://pixogram.co.uk/?page_id=858].

29. [http://www.heritage3d.org/node/105]. 
Knockroe (R4), alors que la surface du monolithe est acquise au moyen d'un scanner Mensi GS101 ${ }^{30}$. Le champ couvert par chaque acquisition étant assez réduit ( $35 \mathrm{~cm}$ x $25 \mathrm{~cm}$ ), il faut donc entreprendre l'assemblage de la mosaïque obtenue (40 scans; fig. 2), l'ensemble du traitement s'effectuant avec le puissant logiciel Geomagic Studio 12. À notre connaissance, sur la foi des images présentées et des résumés d'actions entamées, aucune des démarches engagées à Gavrinis n'a trouvé à Knowth et Knockroe de correspondance et de concrétisation semblable dans l'étude des gravures.

\section{Hollande}

En mai 2010, la société hollandaise Geoscan 3D Media, basée à Baar, proposait un film d'animation autour d'un des rares mégalithes néolithique du pays (D27 à Borger, Drenthe), après avoir effectué un levé lasergrammétrique des orthostates et des dalles de couverture ${ }^{31}$. Mais, ici, aucun cairn ou tertre ne vient perturber la vision animée (très simple) du monument, et aucune gravure à l'intérieur de la chambre n'a nécessité une haute précision du rendu des surfaces.

\section{Allemagne}

Depuis 2005, le Projet "Züschen " développé au sein de l'université de Münster (A. Jockenhövel, D. Dirksen, Lena Loerper), se propose d'enregistrer des gravures sur les parois d'une tombe mégalithique du Néolithique final (Steinkammergrab Züschen I) à l'aide de la profilométrie optique, et d'en restituer une vue tridimensionnelle. L'expérience réussie permet de déceler des superpositions de tracés, notamment une représentation d'attelage de bœufs inédits. Mais une telle manipulation n'est rendue possible que par le transfert de la dalle en laboratoire, ou l'observation d'une réplique en matière synthétique. On note l'emploi des éclairages rasants virtuels pour faire ressortir les tracés ${ }^{32}$ (fig. 1).

\section{Italie}

Assimilés aux sépultures mégalithiques de la façade atlantique, les " temples " de Malte, datés du $\mathrm{III}^{\mathrm{e}}$ millénaire, ne relèvent pourtant pas des mêmes règles architectoniques ni de la même tradition culturelle que les édifices de l'Europe occidentale. L'un d'entre eux, qualifié de la sorte, a cependant bénéficié d'un levé lasergrammétrique par une équipe de tech-

30. SHAW, Rob, DEVLIN, Gary, Laser scanning a decorated orthostat: Knockroe western tomb $R$ 4, Dublin : The Discovery program, 2010, 8 p. [http://www.discoveryprogramme.ie/technology/metric-survey/114-laser-scanning-a-decorated-orthostat-knockroe.html].

31. [http://www.youtube.com/watch?v=3mC8IqXvFLO].

32. [http://www.uni-muenster.de/UrFruehGeschichte/forschen/zueschen.html]. 
niciens, mais davantage pour prouver l'intérêt visuel de la méthode sur des vestiges dont le prestige assure par avance l'attention du public ${ }^{33}$.

\section{Estonie}

Du point de vue historiographique, on prendra soin de rappeler l'emploi de la méthode photogrammétrique (par couples photographiques) appliquée par une expédition estonienne, dès 1986, sur les pétroglyphes gravés par des chasseurs-cueilleurs au creux des affleurements rocheux des bords du lac Onega (Russie), notamment à Besov Nos ${ }^{34}$.

\section{France}

Au milieu des années 90, se sont développées des recherches informatiques visant à travailler sur les propriétés constitutives des objets plutôt que de les approcher par un simple ensemble de coordonnées géométriques. Dit autrement, il s'agissait d'aborder une approche sémantique de la modélisation, en utilisant le langage naturel ou des listes de descripteurs sémantiques. Les objets ainsi créés, chargés de ces descripteurs, pouvaient ensuite interagir de façon cohérente. Les applications sont multiples, allant de la statique des structures à la recherche de dispositifs optimum. Le renversement conceptuel vient de ce que le résultat n'est plus approché par tâtonnement (ou dit plus savamment, par itérations), mais par la réponse souhaitée, le système se chargeant de converger vers le résultat attendu. Un autre changement de paradigme important soutenant la démarche est l'obtention, non pas d'une réponse unique, mais d'un ensemble de solutions dans lesquelles l'utilisateur peut évaluer à la fois ses hypothèses et les résultats obtenus. Le projet MEGAFORM appliqué aux mégalithes - où le plan de Gavrinis fut pris comme idéal-type - avait ainsi pour ambition de " créer rapidement un site mégalithique ".

Le logiciel tente donc, par itération, de s'approcher d'un existant sans chercher, dans un premier temps, à le reconstituer. L'utilisateur décrit tout d'abord un volume englobant, puis ce volume est rempli de pierre (stèles, dalles) dont les formes et volumes sont configurables. L'utilisateur joue dans un système de règles où les volumes maximum et minimum sont définis tout autant que les proportions générales. Les règles sont données par l'observation des sites existants qui donnent les bases du vocabulaire formel. Les dalles et stèles sont ensuite raffinées par la méthode des

33. Baratin, Laura, Checcucci, Giovanni, Curti, S., Lodib, M., Romeo, M., 3-D visualization and animation of architectonic elements for prehistoric megalithic temples of the island of Gozo: the temple of Ggantija, CIPA (International Committee for Architectural Photogrammetry) 2005 XX International Symposium, 26 September - 01 October, 2005, Torino, Italy, 2005, 5 p. http://cipa.icomos.org/fileadmin/template/doc/TURIN/664.pdf].

34. PoikAlainen, Väino, ERnits Enn, Rock Carvings of Lake Onega : the Vodla Region, Tartu : Estonian Society of Prehistoric Art., 1998, 432 p. 
trois ombres (triplombre ${ }^{35}$ ) ou par un vocabulaire descriptif. Si le projet MEGAFORM n'a pas été davantage développé, c'est qu'il présentait à l'utilisateur final, l'archéologue, un paradoxe pénalisant, à savoir reproduire avec moins de détails ce que l'on peut facilement observer et relever sur le terrain. De fait, la question était peut-être mal posée, les enjeux reposant moins sur la recherche d'une forme, que l'on connaît de toute façon, que la dynamique constructive de cette forme ${ }^{36}$. La reprise du projet demanderait d'y intégrer un module de résistance des matériaux et d'avoir en réponse des analyses de contraintes constructives. La modélisation déclarative fait moins parler d'elle désormais, mais pourtant les évolutions des outils numériques font que l'intégration des descripteurs et du langage naturel revient en force.

Nos essais en restitution tridimensionnelle ont débuté en $2003^{37}$, tout d'abord par le biais de la photogrammétrie (couples de photographies numériques calibrées, sur la dalle de chevet de la Table des Marchands) avec l'aide d'un logiciel de l'IGN (mais d'une manipulation lourde et d'un temps de traitement très long), puis par lasergrammétrie sur les monuments de Mané Kerioned à Carnac avec la contribution de la société Mensi (Trimble). Une tentative précédente (1989) avait préconisé un levé photogrammétrique des orthostates et des gravures du monument de la Table des Marchands (photos stéréoscopiques, collaboration DRAC Pays de Loire, Service de l'Inventaire, Nantes), mais faute d'obtenir l'accord des responsables administratifs et scientifiques du site de Locmariaquer, le projet fut arrêté.

Nous débuterons donc avec un premier levé opéré sur le site de Mané Kerioned (Carnac) en utilisant deux scanners à résolution distincte permettant, d'une part, l'enregistrement du site et des architectures (avec le dispositif Trimble GS200, 1 à $5 \mathrm{~cm}$ de résolution), de tester d'autre part une acquisition de gravures sur un orthostate-type (avec un appareil Mensi SOASIC à résolution infra-millimétrique). À ce dispositif lourd et hors de portée du laboratoire à l'époque, sera ajouté un logiciel de photomodéli-

35. Poulet François, Lucas Michel, Modelling Megalithic Sites, "Proceedings of Eurographics' 96 ", Computer Graphics Forum, 1996, Volume 15, Issue 3, p. 279-288.

36. LuCAS Michel, Martin D. et P., PlÉMEnos D., "Le projet ExploFormes, quelques pas vers la modélisation déclarative de formes ", Bigre $\mathrm{n}^{\circ}$ 67, janvier 1990, p. 35-49.

37. Cassen, Serge, Merheb, Moatassem, "Archiving Neolithic heritage ", Paris, 2004 International Conference 3rdDiMENSIon, 2004, p. 13-26. CASSEN, Serge, MERHEB, Moatassem, "Stone surfaces, earth surfaces : notes about the recording and the 3D representation of engraved steles within Neolithic funeral structures in western France (Locmariaquer, Carnac - 4700-3800 cal. BC.) ", In : M. Dobrovolskaya (éd.), Mejedistsplinarie Issledovania v Arkheologii, Interdisciplinary Investigation in Archaeology, Moscou, OPUS, 4, 2005, p. 182191. Cassen, Serge, Lefebvre, B., Merheb M. " Mané Rutual en Locmariaquer (Morbihan). Enregistrement de gravures et simulations architecturales dans une tombe à couloir néolithique ", Revue Archéologique de l'Ouest, 22, 2005, p. 93-124. CASSEn, Serge, Martinez, P., Robin, G., Merheb, M., " Moving the immovable. A short study of methods of recording and illustrating Neolithic engraved steles in Brittany ", dans Virtual Retrospect, Archéologie et réalité virtuelle, colloque international ESTIA/CNRS, nov. 2005, Biarritz, 2006, p. 187-193. 
sation (RealViz) qui permettra la restitution d'orthostates en volumétrie, manipulables dans toutes les directions, quelles que soient la luminosité (souvent faible) et l'incidence lumineuse pour faire ressortir les gravures. Notons que ce type de logiciel est aujourd'hui d'un accès libre alors qu'il coûtait encore une centaine d'euros à l'époque... Ce travail n'a pas encore été publié, et fera prochainement (2014) le point sur l'ensemble des gravures des tombes à couloir concernées.

D'autres essais seront menés en 2005 sur un orthostate de la tombe à couloir du Mané Lud (Locmariaquer), en collaboration avec Philippe Martinez (Laboratoire d'Archéologie, École normale supérieure, Paris) au moyen d'un scanner Konica Minolta VI9i. Mais les nuages de points ne nous resteront pas accessibles, à l'image de la situation vécue pour Mané Kerioned où seul un logiciel de visualisation (RealWorks) permettait de manipuler des nuages réduits en taille (puissance limitée de nos micro-ordinateurs) et d'en extraire des images fixes à basse résolution. Sur la même commune, à partir de 2006, de nouvelles expériences verront le jour à l'occasion du programme d'étude sur le site de la tombe mégalithique Table des Marchands. Un scanning sans miroir à partir d'un tachéomètre permettra de numériser le Grand Menhir ainsi que les monuments du Mané Lud et de Mané Rutual, autorisant des restitutions d'élévations des parois et une visualisation des intérieurs des tombeaux. L'extraction virtuelle des dalles de couverture, anciennes stèles dressées dans l'environnement immédiat, ainsi que la restitution originelle du Grand Menhir brisé, seront des acquis majeurs du programme mené sans véritables moyens financiers. Enfin une démonstration sollicitée en 2006 auprès de la société FARO, dans le cadre d'un diplôme universitaire entamé par C. Bouillon à Nantes, permettra de lever l'ensemble de la Table des Marchands, structures internes et externes. Malheureusement, l'abandon de cette thèse de doctorat pour raisons de santé, et la perte conséquente des données numériques non restituées par le constructeur, ne permettront pas d'achever le travail à peine entamé. Pour sortir de ce mauvais pas et faire aboutir le chapitre consacré aux restitutions tridimensionnelles, un travail reprenant les levés classiques au $1 / 10^{\mathrm{e}}$ (plan et élévations dessinés entre 1986 et 1988) sera mené avec l'aide du logiciel SketchUp, autorisant des simulations architecturales d'ailleurs différentes de la restauration actuelle du site ${ }^{38}$.

En 2009, en concluant le programme sur Locmariaquer, le monument de Gavrinis étroitement lié à la Table des Marchands et probablement un des sites archéologiques les plus visuels de la France de l'ouest, apparut dès lors comme un objet de choix pour reprendre l'exercice de restitu-

38. CASSEN, Serge, GEFFRAY, Olivier, "L'enregistrement 3D des faits accomplis : acquisition de données numériques des volumes sur monument restauré ", dans CASSEN, Serge (dir.), Autour de la Table. Explorations archéologiques et discours savants sur des architectures néolithiques à Locmariaquer, Morbihan (Table des Marchands et Grand Menhir), ACR 20032006, Actes du colloque international, Vannes (Morbihan), 5-7 octobre 2007 (université de Bretagne-Sud, campus Le Tohannic), Nantes, LARA, université de Nantes, 2009, p. 817-825. 
tion tridimensionnelle en vue d'une relecture des signes gravés, gravures prises dans la dimension architectonique de la tombe. Cette fois, la phase de réflexion portant sur les modes d'acquisition va d'emblée associer architectes, géomètres et archéologues pour décider des meilleurs outils; des tests de compilations d'images sous éclairages tournants sont menés sur une céramique de la Table des Marchands, numérisée par scanner, pour lever son décor cannelé à peine visible et le comparer au dessin classique, en comparant également la forme restituée par l'informatique du profil dessiné par le conformateur (fig. 1).

Toujours en Bretagne, un travail récent mené par plusieurs collègues rennais (IRISA, université Rennes 1, CNRS) permet de mettre à disposition des archéologues plusieurs outils et protocoles en vue de la reconstitution de chambres mégalithiques au sein d'un dispositif immersif très performant ${ }^{39}$, une démarche proche de celle adoptée en 2009 pour étudier et visionner Gavrinis sur l'écran Naexus, comme nous le verrons plus loin. L'acquisition s'est faite à partir d'une centaine de clichés numériques traitées sous Agisoft Photo-Scan, et l'usage de l'interface Unity 3D permet à l'observateur/opérateur de naviguer aisément, en temps réel, dans la construction restituée. Il s'agit d'une tombe à couloir réputée dans l'ouest de la France (Carn, Finistère) mais difficile d'accès (caractère insulaire du site, fragilité de l'édifice). Une torche virtuelle pilotée dans Unity est un outil des plus intéressants, qui autoriserait, selon les auteurs, la détection de gravures inédites; mais l'expérience nous apprend que cette méthode d'illumination intuitive est peu efficace à cette échelle si les gravures non pas été détectées au préalable, et leur relevé " archéographique " (dessin vectoriel, chronographie, etc.), quoi qu'il en soit, impossible dans un tel environnement.

En Pays de Loire, dans le cadre d'un projet de maquette virtuelle interactive voulant recréer la vie au Néolithique, Hugues de Lambilly, ancien étudiant en archéologie (Nantes, Rennes), réalise en 2005 (mais avec une mise en ligne en 2011 seulement) une campagne lasergrammétrique sur quelques monuments du littoral vendéen (pierres dressées et tombes à couloir, précision $8 \mathrm{~mm}$ ), à l'aide d'un scanner Minolta Vivid 910. Cette campagne est conçue comme document de promotion dans une recherche de marchés. On relève pourtant une application très démonstrative du procédé d'assemblage de formes numériques sur le site de l'alignement de la Pierre (Avrillé) où l'auteur remarque judicieusement que les deux monolithes bordant la stèle centrale $(5,20 \mathrm{~m}$ hors-sol) sont de taille et de forme similaires, comme deux éléments d'un même bloc, ce que n'avait pas remarqué le fouilleur du site ${ }^{40}$.

39. Gaugne, Ronan, Barreau, J.-B., Cousseau, F., Gouranton, V., " Large-scale immersive reconstitution of a Neolithic corbel dôme ", dans The $13^{\text {th }}$ International Symposium on Virtual Reality, Archaeology and Cultural Heritage VAST (2012) D. Arnold, J. Kaminski, F. Niccolucci, and A. Stork (Eds), 2012, 8 p. [http://hal.archives-ouvertes.fr/hal-00748894].

40. Beneteau, Gérard, "L'alignement de menhirs de "La Pierre" à Avrillé (Vendée) ", Bulletin de la Société préhistorique française, tome $90, \mathrm{n}^{\circ}$ 2, 1993, p. 151-158. Voir : [http:// patrimoine3d.blogspot.fr/2011/05/un-bloc-pour-deux-menhirs.html]. 
Figure 1 - Photogrammétrie de la tombe à couloir de Anta Grande de Zambujeiro, Portugal (d'après Soares, Tavaraes da Silva 2010); lasergrammétrie de Stonehenge (d'après Abbott, Anderson-Whymark 2012); profilométrie optique sur des orthostates gravés de Züschen I, Allemagne (d'après Jockenhövel et al. 2005); lasergrammétrie et remontage virtuel des blocs dans l'ouvrage de stèles de La Pierre à Avrillé (d'après Lambilly 2011); lasergrammétrie d'une poterie Castellic du sol enterré sous la Table des Marchands à Locmariaquer (d'après

Grimaud, thèse en cours).

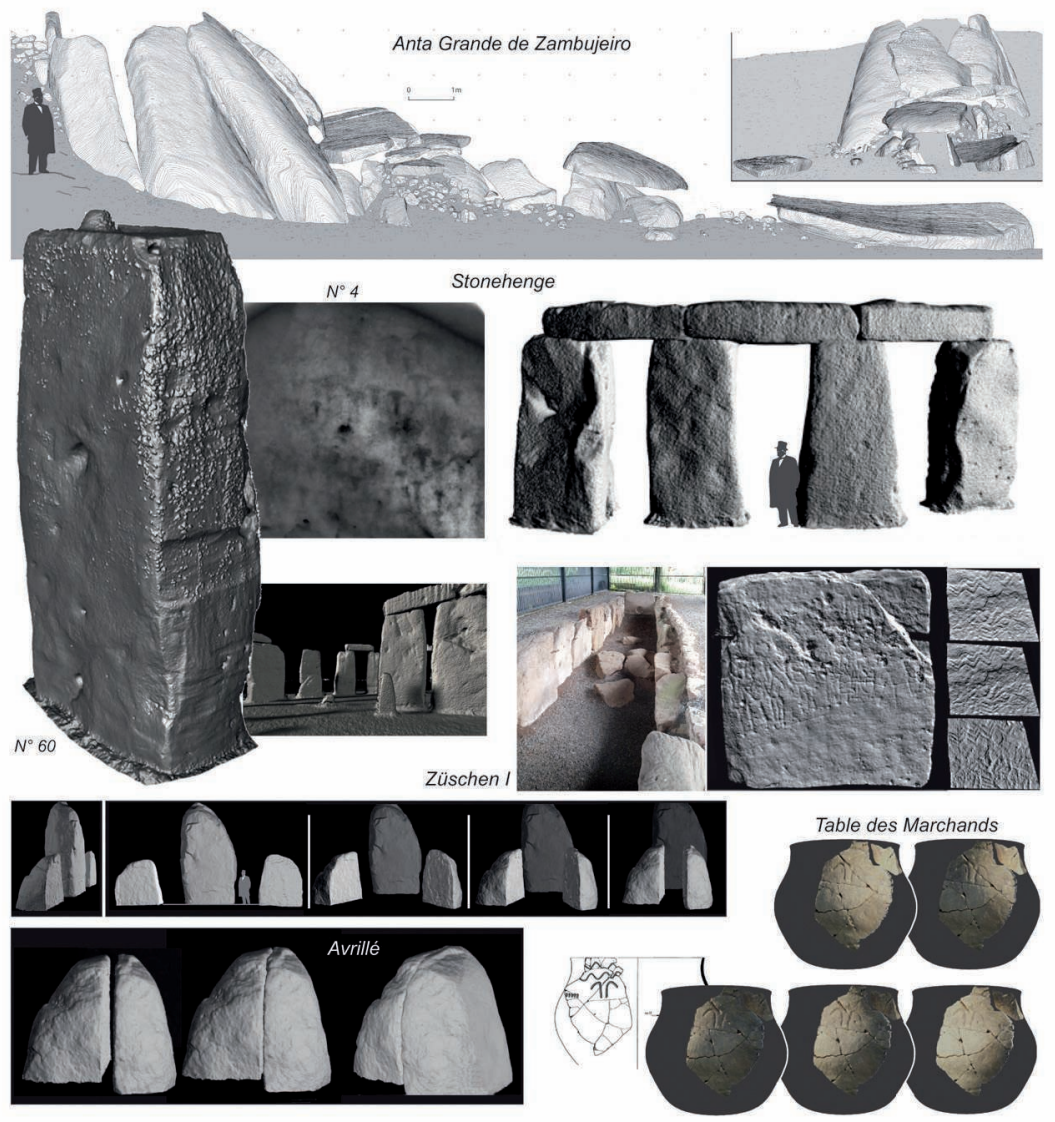

La manipulation des deux formes permet de confirmer l'hypothèse : les deux stèles ne formaient à l'origine qu'un seul bloc, une veine de quartz au cœur du granite étant mise à profit pour le partager (fig. 2 - planche couleur). Cette manière de rapprocher virtuellement des modèles numériques de terrain (MNT) nous avait permis en Morbihan de raccorder deux 
fragments d'une même stèle sur le site du Vieux Moulin à Plouharnel en validant l'hypothèse de départ ${ }^{41}$.

Un atelier 3D organisé à Nantes par Yann Le Jeune du SRA des Pays de Loire (22 mai 2012) sera de même l'occasion de démontrer l'utilité pratique des levés photogrammétriques à partir d'un simple appareil photo numérique (APN). La tombe à couloir de la Planche à Puare (lle d'Yeu, Vendée) servira d'objet de référence. Un premier modèle, généré avec le logiciel Photoscan à partir de 150 photographies, est bel et bien opérationnel mais l'orthoréférencement nécessitera l'intervention de micro-ordinateurs bien plus puissants que nos unités centrales de bureau (coll. Stéphane Jupin) étant donné le poids des fichiers. La volonté de traiter les nuages de points au sein d'un SIG (système d'informations géographiques) ${ }^{42}$ permet de créer rapidement une bonne documentation en plans horizontaux des volumes du site. Cependant, la recherche d'informations plus précise telles des gravures se trouvera limitée à cause de la perte de la troisième dimension qui semble inhérente à tous les logiciels de SIG. Les sections ou coupes architecturales semblent actuellement hors d'atteinte avec ces outils.

Plus au sud, en Poitou, un des tumulus de Péré à Prissé-la-Charrière (Deux-Sèvres) a récemment fait l'objet d'un levé lasergrammétrique mais l'information extraite des nuages de points se limite pour l'instant à quelques illustrations générales ${ }^{43}$.

Enfin en France méridionale, sur la commune de Saint-Paul-le-Jeune (Ardèche), un inventaire des sépultures mégalithiques a permis dès 2002 quelques restitutions tridimensionnelles réalisées par la société ATM.3D. Les arêtes des monolithes sont levées au tachéomètre à mesure laser sans prisme. Un fichier cartographique des lignes filaires 3D codées a ensuite été généré à partir du fichier brut de levé enregistré dans l'appareil topographique. Chaque modèle 3D filaire a ensuite été projeté dans 5 vues orthographiques pour produire une cartographie au 1/50e. Les résultats ont été fournis au format Acrobat. À notre connaissance, aucune utilisation archéologique n'est décrite en prolongement de cette expérience ${ }^{44}$.

\section{La restauration des tombes a couloir de la France de l'Ouest : étude de cas à la Table des Marchands}

Les raisons et motivations qui conduisent à la restauration des monuments mégalithiques ne sont pas le sujet de ce chapitre. Elles peuvent être conservatoires, identitaires, patrimoniales, commerciales, pédagogiques,

41. Cassen, Serge, Vaquero Lastres, Jacobo, Les Marches du Palais, Recherches archéologiques sur alignements de stèles et tertres funéraires néolithiques autour de la baie de Quiberon (Morbihan, 2000-2002), Nantes, Laboratoire de Préhistoire, université de Nantes, 2003, 166 p.

42. [http://www.atelier3d.levillage.org/spip.php?article22].

43. Laporte, Luc, Scarre, C., Joussaume, R., Soler, L., " Nouvelles données sur le mégalithisme de l'ouest de la France ", L'Archéo-théma 10, 2010, p. 7-12.

44. [http://chazaly.pagespro-orange.fr/html/dolmens.htm]. 
etc., séparément ou tout à la fois... Le principe qui préside au schéma architectural adopté depuis plus de cinquante ans et la critique de cet état de fait forment au contraire le fond de notre discussion. La représentation tridimensionnelle servira d'entrée à la problématique.

En posant à part les ensembles restaurés depuis la fin du XIX ${ }^{\mathrm{e}}$ siècle jusqu'aux années 1930, qui conduisent le plus souvent en Bretagne à redresser des supports, à rapporter des dalles de couverture manquantes, à ramener les éboulis au centre de l'enveloppe originelle sans vraiment tenir compte des murs externes de limitation (travaux de Z. Le Rouzic, pour ne s'en tenir qu'à une figure emblématique en région carnacoise), il ne fait guère de doute que le cairn à chambres multiples de Barnenez (Finistère) est la figure archétypale du monument mégalithique telle que la communauté scientifique se la représente à partir du milieu des années 60 (fig. 3). Autrement dit le cairn à gradins ou à degrés. Il est difficile de dire qui, de P.-R. Giot ou de son élève J. L'Helgouac'h, est à l'origine de cette image convenue qui servira de modèle à tous les monuments restaurés à partir dès années 1960, qu'il s'agisse de la Bretagne, de la Normandie, de l'Anjou, de la Vendée ou du Poitou (fig. 4). Il est en tout cas certain que, dans sa thèse, J. L'Helgouac'h énonce et figure de façon assez novatrice comment doit se constituer un cairn-type à enceinte, chaque mur concentrique délimitant une terrasse horizontale ${ }^{45}$ (fig. 3; L'Helgouac'h 1965, p. 27 fig. 6), tandis que dans le même temps Barnenez est en cours de restauration.

\section{Figure 3 - Barnenez (Finistère), photographies du monument actuel et du chantier lors de sa restauration (photos $S$. Cassen et P.-R. Giot); vue isométrique d'un modèle de cairn à degrés (d'après L'Helgouac'h 1965).}

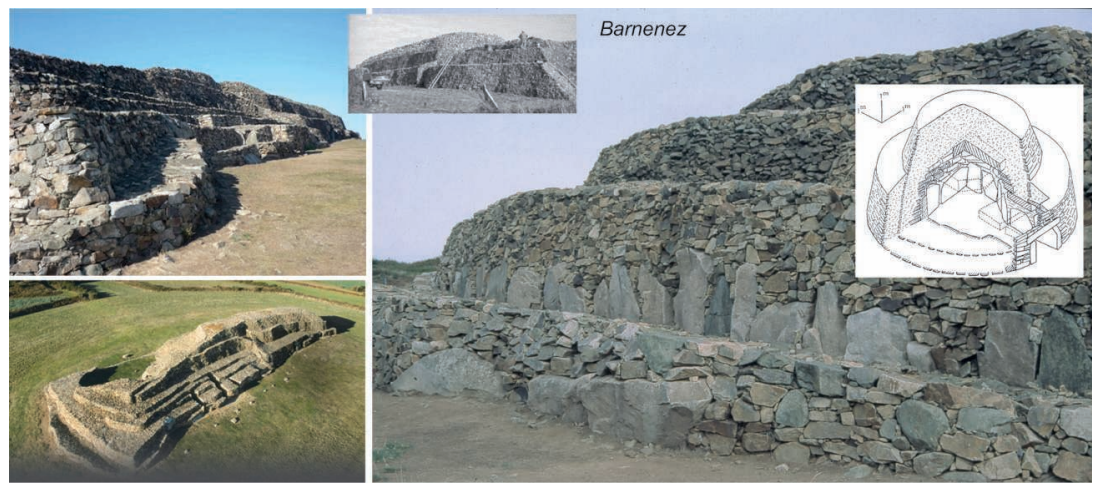

45. L'Helgouac'H, Jean, Les Sépultures mégalithiques en Armorique, Rennes, université de Rennes, 1965, 331 p. 
Pour une critique de la représentation tridimensionnelle des architectures...

Figure 4 - Cairn à degrés des tombes à couloir restaurées de Bougon (Deux-Sèvres), Petit Mont (Morbihan) et Les Mousseaux (LoireAtlantique) (photos S. Cassen).
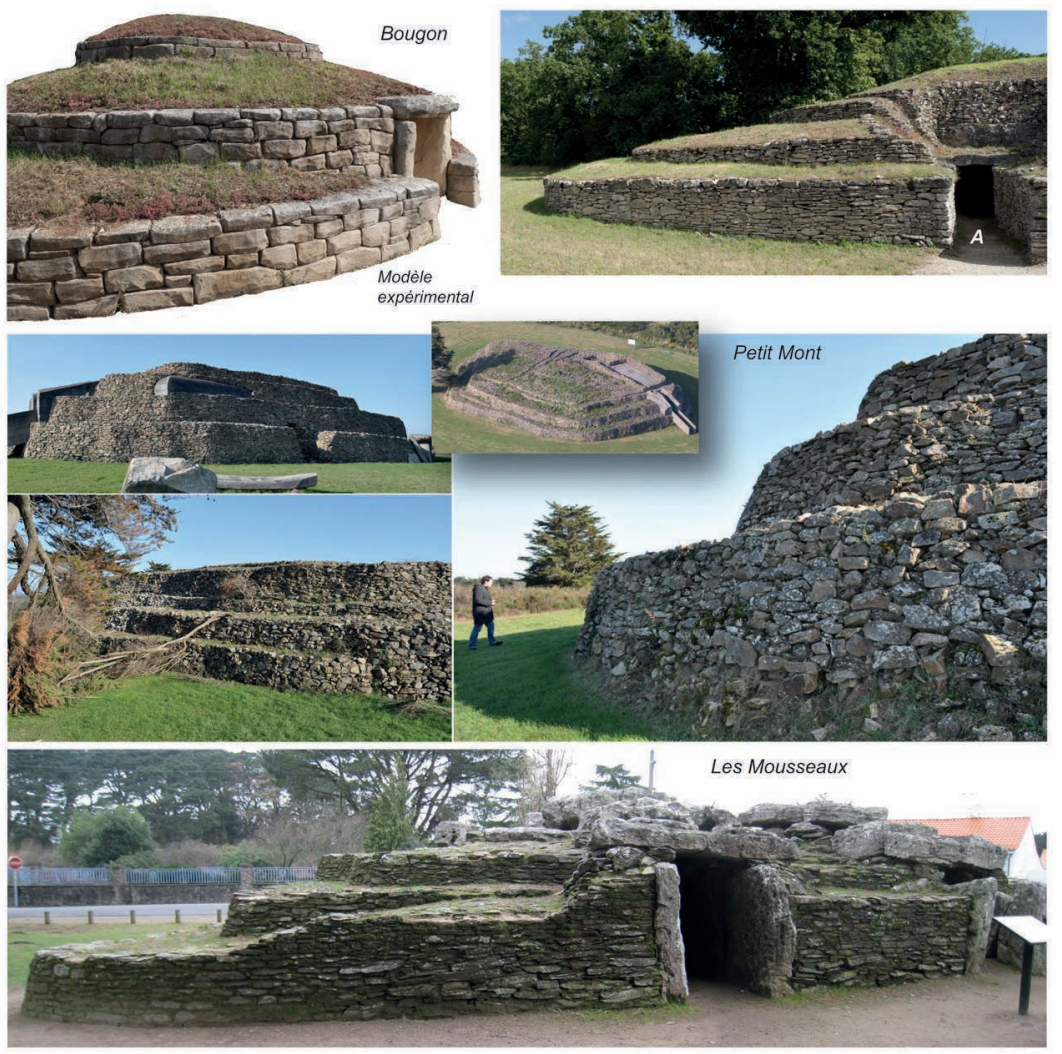

Ce parti-pris provient certainement d'une reconnaissance assez tardive des archéologues de la France de l'Ouest relativement aux appareillages structurant le cairn ou le tumulus, non seulement au sein de la masse des pierres et sédiments accumulés autour de l'espace sépulcral, mais plus encore en façade et périphérie du monument ainsi constitué. C'est en effet tout le mérite de nos collègues œuvrant sous l'impulsion de P.-R. Giot d'avoir reconnu, défendu et revendiqué ces éléments d'architecture, juste entrevus dans le passé (notamment à l'lle Longue par Z. Le Rouzic). Mais on peine en revanche à comprendre les arguments qui vont conduire à l'image du cairn à gradins que nous connaissons désormais et qui semble s'imposer sans discussion.

La pétition de principe adoptée par J. L’Helgouac'h est assez claire : « Il est certain [...] que les enceintes, quadrangulaires ou circulaires, n'étaient pas internes dans la masse du cairn, mais visibles, étagées en paliers. 
Leur rôle était à la fois de maintien de la masse des pierres et d'esthétique architecturale $^{46}$. " On chercherait en vain, dans les coupes stratigraphiques levées au cours des fouilles de mégalithes par notre collègue, la preuve graphique ou photographique d'une telle assertion. Un retour à la publication de Barnenez s'impose, malgré là encore un défaut alarmant de relations stratigraphiques. Parmi une abondante production, aucun autre article de P.-R. Giot ne donne en effet d'explication au choix de cette représentation des élévations, d'où suivra la restauration.

Le premier constat établi par le fouilleur de Barnenez est un manque de comparaison à l'échelle européenne ${ }^{47}$, faute d'y connaître de cairn intact. On peut néanmoins se remémorer - outre le cairn de l'lle Longue déjà cité et restauré en 1914 - les monuments fouillés en Irlande au milieu des années cinquante, connus de P.-R. Giot à l'époque où il entreprend ses explorations et premières restaurations, qui non seulement contiennent une structuration interne de l'enveloppe pierreuse mais présentent des essais graphiques de restitution tridimensionnelle qui laissent une forme courbe à l'ensemble de la couverture, sans visibilité de cette division interne, seulement limitée en périphérie par un parement de faible hauteur ${ }^{48}$. Et dix ans avant que ne paraisse la monographie de Barnenez, mais dix ans après la fin des différentes restaurations du monument, la proposition graphique du grand cairn de Quanterness en Écosse, à murets concentriques circulaires autour de la tombe à couloir, ne suppose aucun gradin successif mais au contraire une forme arrondie du cairn en accord avec le contexte stratigraphique $^{49}$ (p. 66, fig. 32).

Par défaut, donc, on imagine à Barnenez " que le sommet était sensiblement plan et horizontal ${ }^{50}$ ". Quant aux flancs du cairn, on décide de monter les différents murets reconnus jusqu'au niveau maximum où ils se trouvaient attestés en un endroit de leur développement, ce qui semble une décision tout à fait défendable, en stockant les matériaux en retrait des murets de parements internes, déterminant de facto la formation d'une terrasse, ce qui est cette fois une création architecturale. Mais l'auteur ajoute que cette manière de faire " ne vise qu'à donner une idée du volume et de l'altitude maximale, sans préjuger du profil du sommet des parements. Il est vraisemblable que ceux-ci continuaient avec leur fruit qu'on leur connaît ${ }^{51} "$.

46. Ibid. p. 24.

47. Giot, Pierre-Roland, Barnenez, Carn, Guennoc, Rennes, Travaux du Laboratoire d'Anthropologie Préhistoire, université de Rennes I, 1987, 232 p., p. 88.

48. O'KeLLY, Michael, "A wedge-shaped gallerygrave at Island, Co. Cork ", The Journal of the Royal Society of Antiquaries of Ireland, Vol. LXXXVIII, 1958, p. 1-23. O'Kelly, Michael, "A horned cairn at Shanballyedmond, Co. Tipperary ", Journal of the Cork Historical and Archaeological Society, 63, 1958b, p. 37-72.

49. RenFrew, Colin, AitKen, M. J., Judson, C.T., Investigations in Orkney, London, Society of Antiquaries of London, Thames and Hudson, 1979, $234 \mathrm{p}$.

50. Giot, P.-R., op. cit., 1987, p. 88.

51. Ibidem, p. 89. 
Le point de vue de P.-R. Giot est par conséquent beaucoup moins assuré que celui de J. L'Helgouac'h quant à l'existence de paliers visibles; c'est pourtant la seconde opinion qui prévaudra. Ceci ne saurait porter à longue discussion si le caractère didactique de la restauration primait en mettant en évidence ces murets internes (les restaurations menées à Bougon A et F1 sont à cet égard un choix intermédiaire recevable, mais elles sont beaucoup plus discutables au-dessus du dolmen F0 - fig. 4). Ces renforcements successifs de la masse des matériaux au fur et à mesure de l'avancement des travaux, ces étapes de la construction qui peuvent marquer autant de phases chronologiques bien distinctes, ces lignes géométriques qui peuvent aussi relever de préoccupations symboliques à l'image d'un plan dessiné au sol, sont pêle-mêle des éléments du discours qui peuvent bien entendu déterminer cette volonté de restauration en forme de pyramide égyptienne ou méso-américaine. Mais on ne saurait confondre le principe de « l'escalier " développé par ces dernières sociétés ${ }^{52}$, et la structure adossée par accrétion propre aux cairns néolithiques. Le principe de l'escalier sous-tend à la fois la structure à degrés adossés, le processus de sa mise en œuvre sur chantier et le principal procédé de levage des blocs, sans compter le symbole égyptien de l'ascension du défunt par marches successives depuis le monde souterrain vers un au-delà dans le ciel, ou encore les différents niveaux du monde inférieur représentés par les gradins de la pyramide Maya au surplus munie d'escaliers réels dirigés vers le temple sommital. On ne peut aujourd'hui attester, par une démarche scientifique rationnelle, le schéma revendiqué pour le Néolithique de l'Ouest, imposé à tous, et l'on sait par contre combien une certaine littérature ésotérique a beau jeu de s'emparer de telles analogies à longue distance pour entretenir tel ou tel mystère.

Une des conséquences de ce parti-pris est parfaitement illustrée par la restauration du monument emblématique de la Table des Marchands dont une première critique fut énoncée, d'autant plus légitime que nous y avions passé neuf années en travaux de fouilles ${ }^{53}$. Cette tombe à couloir est en effet emblématique à plus d'un titre, pour avoir été tout d'abord l'éponyme fameux choisi en 1796 à partir du toponyme local dolmin, pour avoir été ensuite le premier "dolmen " morbihannais à être fouillé en $1811^{54}$. Ces

52. GuERrier, Éric, Pyramides ou le principe de l'escalier, Paris, L'Harmattan, 2012, 288 p.

53. CASSEn, Serge, "La simulation des faits imaginés : phases, séquences, scénarios historiques autour d'une barre de stèles et d'une tombe à couloir ", dans Autour de la Table. Explorations archéologiques et discours savants sur des architectures néolithiques à Locmariaquer, Morbihan (Table des Marchands et Grand Menhir), Actes du colloque international, Vannes, université de Bretagne-Sud, 5-7 octobre 2007, Nantes, université de Nantes, LARA, 2009, p. 881-909.

54. Chaigneau, Cyrille, Cassen, S., Le Gaudion, Y., "L'historiographie en Locmariaquer, Une première approche ", In : Autour de la Table. Explorations archéologiques et discours savants sur des architectures néolithiques à Locmariaquer, Morbihan (Table des Marchands et Grand Menhir). Actes du colloque international, Vannes, université de Bretagne-Sud, 5-7 octobre 2007, Nantes, université de Nantes, LARA, 2009, p. 387-417. 
raisons sont suffisantes pour que nous prenions soin de l'image véhiculée (voir, à ce sujet, la critique faite par F. Carrera 2011b) ${ }^{55}$.

Et ce fut d'ailleurs à travers un effort de représentation tridimensionnelle que le volume de l'enveloppe fut questionné (fig. 5 - planche couleur). Les rapports stratigraphiques permettant d'évaluer le volume des éboulis et leur pente, et donc la hauteur présumée à l'aplomb de la structure interne, témoignent indubitablement d'un défaut de recouvrement de la dalle spectaculaire placée sur la chambre funéraire. Une simple comparaison avec le cairn de Gavrinis permet de s'en convaincre, où la dynamique des dépôts au-delà des parements périphériques n'est pas du tout superposable à celle relevée à la Table des Marchands. Il n'est pas question ici de cairns plus ou moins amputés au cours du temps, puisque justement la périphérie de ces masses fut la mieux préservée des carriers et la plus lisible en stratigraphie.

La restauration interne pose également problème et s'éclaire tout autant à l'aide d'une représentation tridimensionnelle (fig. 6 - planche couleur). Plusieurs faux orthostates en ciment ont en effet été replacés dans la chambre, soit au-dessus d'un chicot de support brisé découvert en fouille, soit à l'emplacement d'un calage préservé laissant en négatif la forme basale de l'orthostate. Ces substitutions ne sont pas très critiquables en soi, bien que les morphologies adoptées soient bien sûr tout à fait gratuites (on peut tout autant se contenter de signaler discrètement les manques dans la paroi, dus aux destructions). Mais l'envergure choisie des supports n'est malheureusement pas en accord avec le résultat archéologique et déforme le projet architectural initial. Le pilier $\mathrm{n}^{\circ} 8$ est à cet égard fort démonstratif : poussé jusqu'à toucher le plafond, il est en réalité tangent à cette dalle, sans être porteur, et un déplacement en temps réel dans la structure en 3D permet de s'en assurer. Il est donc inutile et faux, et la seule prise en compte des orthostates bien plus réduits qui l'encadrent (les $\mathrm{n}^{\circ} 7$ et 9, intacts) suffit à faire comprendre cette faute de restauration.

On voit, en somme, combien le poids des " visuels " préconçus, un argument d'autorité et un plan rigide et prématuré de la restauration ont pu abîmer une image. Image archétypale certes, mais au point inconcevable de l'effacer de l'ethnographie d'un lieu (la " Table " a disparu).

\section{Perspectives}

Le programme de recherches en cours depuis 2009 sur le monument de Gavrinis, qui prend en compte d'autres gisements pour compléter l'environnement méthodologique (stèles de Kermaillard, Kermarquer, Kerjouanno...), tente de répondre à plusieurs des questions et critiques soulevées dans les paragraphes précédents. Comment rendre compte d'un

55. CARRERA RAmírez, Fernando, "Crónica del fracaso. Tipos y criterios de intervención en monumentos megalíticos ", dans Primer congreso de prehistoria de Andalucía : la tutela del patrimonio prehistórico, Antequera 22-25 de septiembre de 2010, 2011, p. 419-432. 
état architectural ancien, originel s'il en est, à partir des données de l'archéologie? Comment stabiliser le savoir pour mieux le transmettre à un moment donné, malgré la somme des incertitudes et des doutes inhérents à la méthode d'investigation et à la profondeur chronologique?

Pour se donner de nouveaux moyens de représentation et tenter l'autre terme de l'alternative - celui qui propose une restauration réversible dans le temps d'une génération -, l'acquisition de données numériques des surfaces des objets devient un passage obligé. Le but technique est de pouvoir recomposer des volumes à partir de ces surfaces, d'en extraire des mesures exactes; le but archéologique est de les présenter à notre vue dans l'ordre architectural actuel afin d'en inférer un ordre hypothétique à l'époque néolithique, en testant des hypothèses structurales, historiques, architectoniques. Et non seulement il importe à Gavrinis de travailler à l'échelle du cairn pour en restituer la morphologie, mais encore de traiter le tracé gravé sur la paroi du tombeau car tout ce jeu esthétique des reliefs mérite ici aussi d'être virtuellement éclairé pour en extraire le signe.

On le sait, une grande part de l'émotion ressentie lors de la visite de Gavrinis (dont une succession de transitions préalables n'est pas sans participer au phénomène ressenti : le passage en barque, la montée depuis la plage vers le sommet de l'île, le passage de la lumière à l'obscurité) tient à la profusion des gravures couvrant les parois du couloir et de la chambre funéraire. À tel point que l'on discute parfois la seule fonction funéraire pour suggérer l'idée d'un temple ou sanctuaire; peu importe dans l'immédiat. Ces tracés innombrables doivent pouvoir être identifiés, puis qualifiés. Un enregistrement lasergrammétrique et photographique vient d'en constituer un corpus inédit, grâce auquel le relief de la roche participe enfin à la lecture des signes, tandis que la chronologie recomposée des tracés contribue à restituer l'ordre d'apparition des motifs (fig. 7 - planche couleur). Cette chronographie est d'ailleurs un travail de longue haleine, où l'éclairage virtuel en surface des dalles, par son apparente facilité d'exécution, suppose néanmoins une observation attentive et un contrôle croisé des moyens de restitution du relief.

Tout le travail à venir devra rendre compte de cette accumulation de gravures dans un système cohérent de représentation, d'en faire ressortir le sens topographique de la réalisation, d'en inférer un sens topologique en qualifiant des entités que nous nommons sémiotiques pour bien faire comprendre l'intention de départ. Ces signes racontent une histoire en surface d'une dalle, et peut-être en passant d'une dalle à l'autre dans une direction qu'il nous appartiendra de trouver, peut-être même dans un autre ordre architectural, différent de celui que nous enregistrons qui n'est qu'un état terminal. Cette information touffue et déployable à différents niveaux d'exposition, trouvera (et trouve déjà à travers un prototype exécutable) un support idéal de projection dans le dispositif immersif Naexus (fig. 7) développé par l'École nationale supérieure d'architecture de Nantes et l'école d'architecture de Dessau en Allemagne (écran circulaire). De la visite vir- 
tuelle passive à la maîtrise individuelle d'un " déplacement " en temps réel, seront donnés à voir et à comprendre ce qu'il ne sera jamais possible de restituer à une telle échelle sur le site, et avec une telle interactivité (sur le terrain, bien entendu, le visiteur pourra être munis de son téléphone mobile ou d'écrans tactiles propres à déclencher, au passage de telle ou telle composition, l'explication temporaire des archéologues).

Il en sera de même du volume architectural d'ensemble, qui devra rendre justice des expressions employées à dessein par les archéologues à Barnenez, Table des Marchands ou Gavrinis (murs en " pelure d'oignons ", placage "d'écailles " de pierres ${ }^{56}$ ) - expressions qui rendent compte de la construction vraiment observée sur le terrain -, pour suggérer une autre alternative au cairn à degrés en laissant au spectateur de passage un choix parmi d'autres possibles.

Chaque être humain, chaque peuple, selon ses fins, écrivait F. Nietzsche, a besoin d'une certaine connaissance du passé, tantôt sous forme d'histoire monumentale, tantôt sous forme d'histoire antiquaire (" traditionnaliste", dirions-nous aujourd'hui), tantôt sous forme d'histoire critique, « mais non point comme en aurait besoin une troupe de purs penseurs qui ne fait que regarder la vie, non comme des individus avides de savoir et que seul le savoir peut satisfaire, pour qui l'augmentation de la connaissance est le but même de tous les efforts, mais toujours en vue de la vie [... $]^{57}$ " (chap. 4). Car l'histoire appartient au vivant sous trois rapports, parce qu'il est actif et qu'il aspire, parce qu'il conserve et qu'il vénère, parce qu'il souffre et qu'il a besoin de délivrance. À cette trinité de rapports correspondent le point de vue monumental, le point de vue antiquaire et le point de vue critique.

Ce n'est pas la moindre des tâches de l'archéologue et du médiateur que de parcourir ces trois espèces d'histoire au sein des rapports sociaux, en recherchant la plus grande proximité de l'autre à travers les millénaires, notamment par la perception commune de ces fondamentaux que sont l'eau et la roche, et leur traduction métaphorique, esthétique, phénoménologique. Et non pas seulement en cherchant une idée générale et le thème final, mais en paraphrasant spirituellement un thème connu, peut-être ordinaire, une mélodie de tous les jours, ajoute F. Nietzsche, pour l'élever jusqu'au symbole universel afin de laisser entrevoir, dans le thème primitif, tout un monde de profondeur et de beauté, la signification même de l'histoire. Ce n'est donc que par la force du présent, conclut le philosophe, que doit être interprété le passé; ce n'est que par la plus forte tension de nos facultés les plus nobles que nous devinerons ce qui est grand. Autrement nous abaissons le passé à notre niveau.

56. LE Roux, Charles-Tanguy, Gavrinis et les îles du Morbihan, Guides archéologiques de la France, Paris, ministère de la Culture, 1985, 96 p.

57. Nietzsche, Friedrich, Seconde considération intempestive, De l'utilité et des inconvénients de l'histoire pour la vie, (Trad. Henri Albert), Paris, Flammarion, collection GF, $\mathrm{n}^{\circ} 483,1874$ (1988), $187 \mathrm{p}$. 


\section{RÉSUMÉ}

L'objectif de cet article est de rendre compte des moyens employés pour décrire et analyser non seulement une architecture mégalithique mais encore les signes gravés et peints en surface des parois, tous susceptibles de donner un accès privilégié à la pensée des Ve et IVe millénaires av. J.-C. Le but technique est de recomposer des volumes à partir de ces surfaces, et d'en extraire des mesures exactes; le but archéologique est de les présenter à notre vue dans l'ordre ou le désordre architectural actuel afin d'en inférer un ordre hypothétique à l'époque néolithique, en testant des hypothèses structurales, historiques, architectoniques. La réflexion menée sur les diverses acquisitions des données spatiales et la représentation tridimensionnelle de ces monuments permettra de revenir sur une question inhérente à la représentation, qui est celle de la restauration, en évoquant des expériences européennes et armoricaines, notamment au sein des tombes à couloir de Gavrinis et de la Table des Marchands en Morbihan.

\section{ABSTRACT}

This article seeks to clarify the means used to describe and analyse not only megalithic architecture but also the signs engraved and painted on their surfaces that give privileged access to human thought in the 5th and 4th millennia BC. The technical purpose is to reconstitute volumes from these surfaces, and to extract exact measurements. The archaeological purpose is to present them to us in their current architectural order or disorder to deduce a hypothetical order in the Neolithic period, by testing structural, historical and architectonic hypotheses. The discussion of diverse methods of spatial data collection and the three-dimensional representation of these monuments will enable us to return to a question inherent to the representation, namely that of restoration, by evoking European and Armorican experiences and, in particular, the passage graves of Gavrinis and La Table des Marchands in Morbihan. 